\title{
Ecocrítica y resistencia pedagógica: formación docente y contextos de encierro
}

\author{
ECOCRITICAL AND PEDAGOGICAL RESISTANCE: TEACHER TRAINING AND \\ CONFINEMENT CONTEXTS
}

\section{ECOCRÍTICA E RESISTÊNCIA PEDAGÓGICA: A FORMAÇÃO DOCENTE E CONTEXTOS DE RECLUSÃO}

\author{
Jimena Marrero Cruz ${ }^{* 1}$ \\ Ximena Abitante ** \\ María Dipaola ** \\ jimenamarrero@cerpsur.uy \\ abitantex@gmail.com \\ mariagabrieladipaola@gmail.com
}

\section{Resumen}

El artículo presenta la reflexión y la experiencia en torno a la línea de pensamiento pedagógico que fundamenta un proyecto de extensión en formación del profesorado. Se abordan desafíos que vinculan trabajo manual con trabajo intelectual, desde la praxis pedagógica crítica. Nos basamos en la metodología extensionista crítica, la cual entendemos como un espacio co-construído de cooperación entre los centros educativos y otros sectores de la sociedad de la que forma parte. La metodología de trabajo extensionista se acciona desde la resistencia ecocrítica, trabajando lo educativo desde aquellos elementos que promueven los saberes comunitarios. Entendemos que el trabajo que se llevó a cabo entre colectivos del "Almendro Centro Cultural" en la Unidad $\mathrm{N}^{\circ} 4$ del Instituto Nacional de Rehabilitación (INR) Santiago Vázquez y la "Usina Cultural Matices" en la Unidad N6 del INR de Punta de Rieles, durante dos años (2017/2018), es superar las lógicas de encierro por diferenciación o segregación, desde la reflexión y la acción. Concluimos sobre la posibilidad de construcción del saber ecopedagógico crítico, desde la alteridad de la producción de un espacio educativo que no puede ser comprado ni vendido.

Palabras clave: ecopedagogía, ecocrítica, formación del profesorado, contextos de encierro

\footnotetext{
1 * Consejo de formación en educación. Administración nacional de Educación pública. Consejo directivo central.

** Centro regional de profesores del sur.

Tekoporá ${ }^{\circledR}$. Centro Universitario de la Región Este. Universidad de la República (C) Marrero, et al. (2021)

Este es un artículo de Acceso Abierto distribuido bajo licencia Creative Commons (CC BY NC 4.0)
} 


\begin{abstract}
This article presents experience and reflection around the pedagogical line of thought underlying an extension project in teacher training. Challenges that link manual work with intellectual work are addressed from the angle of critical pedagogical praxis. Our praxis is based on critical extension methodology, which we understand as a co-constructed space of cooperation between educational centers and other sectors of the society of which they are a part. The extension work methodology operates from a place of ecocritical resistance, working with educational elements that promote community knowledge. We understand that the work carried out between groups of the "Almendro Cultural Center" in Unit $N^{\circ} 4$ of the Santiago Vázquez National Institute of Rehabilitation (INR) and the "Usina Cultural Matices" in Unit $N^{\circ}$ 6 of the INR of Punta de Rieles, for two years (2017/2018), succeeded in overcoming the logics of confinement by differentiation or segregation, from a place of reflection and action. We suggest the possibility of constructing critical eco-pedagogical knowledge, from the production of alternative educational spaces that cannot be bought or sold.
\end{abstract}

Keywords: ecopedagogy, ecocriticism, teacher training, confinement contexts

\title{
Resumo
}

O artigo apresenta a reflexão e a experiência em torno do pensamento pedagógico que fundamenta um projeto de extensão na formação de professores. São abordados os desafios que vinculam o trabalho manual ao trabalho intelectual, a partir da práxis pedagógica crítica. Nos baseamos na metodologia de extensão crítica, que entendemos como um espaço coconstruído de cooperação entre centros educacionais $e$ outros setores da sociedade da qual faz parte. A metodologia de trabalho de extensão é operada a partir da resistência ecocrítica, trabalhando a educação a partir daqueles elementos que promovem o conhecimento da comunidade. $A$ experiência foi sistematizada a partir do olhar coletivo corporificado nos roteiros. Entendemos que o trabalho realizado entre grupos do Centro Cultural Almendro "na Unidade $N^{\circ} 4$ do Instituto Nacional de Reabilitação Santiago Vázquez (INR) e a "Usina Matices Culturais" na Unidade $N^{\circ} 6$ do INR de Punta de Rieles, ao longo de dois anos (2017/2018), é superar as lógicas do confinamento pela diferenciação ou segregação, da reflexão e da ação. Concluímos sobre a possibilidade de construção de saberes ecopedagógicos críticos, a partir da alteridade da produção de um espaço educacional Não pode ser comprado ou vendido.

Palavras-chave: ecopedagogia, ecocrítica, formação de professores, contextos de reclusão

\section{Introducción}

El tema que nos convoca en el presente artículo refiere a la experiencia concreta de vínculo pedagógico de enseñanza y aprendizaje entre personas privadas de libertad ambulatoria (PLA) y estudiantes y profesora del Centro 
Regional de Profesores del Sur "Clemente Estable" (CeRP del Sur). Surge la inquietud por abordar una práctica concreta con las personas PLA por ser potenciales futuros estudiantes de los futuros profesores que egresan de la formación docente. El espacio áulico donde se comenzó a gestar la necesidad de involucrarnos con los contextos de encierro fueron los cursos de Pedagogía 1 y de Observación y Análisis de las Instituciones Educativas, ambos pertenecientes a la currícula formal de la formación del profesorado y dictados por una de las autoras presentes. En dichos cursos la temática "contextos de encierro" se trabaja desde un enfoque pedagógico crítico-reflexivo, principalmente por entender que es necesario que esté presente en la carrera, pero identificando que está ausente en los programas oficiales. Por otro lado, en el intercambio docente-estudiantes, se revela la necesidad de desmitificar lo teórico y llevar adelante formas prácticas de involucramiento $\mathrm{y}$ aprendizaje mutuo con las personas PLA habitando los contextos de encierro.

Asimismo, hasta el presente se trabajan a lo largo de los cursos nociones de pedagogía permacultural, que abordan epistemologías de la educación referentes a una ecocrítica y resistencia pedagógica. Llevar por este camino los contenidos de la experiencia nace del diálogo entre estudiantes y docente junto con personas privadas de libertad ambulatoria (PLA) respecto a qué temas/contenidos abordar, y de un proyecto de huerta del CeRP del Sur en el año 2015. Por otro lado, tomando como punto de partida el proceso de transformación de la formación en educación hacia la Universidad de la Educación, pensamos en el problema de la extensión en la formación inicial del profesorado, surgiendo así un análisis sobre cómo se lleva a cabo, cuáles son sus bases y sus consecuencias.

La acción extensionista entiende necesario, desde el inicio de la elaboración del proyecto, problematizar la producción del alimento, su distribución y consumo. En este sentido, la educación centrada en la autoproducción tiene particular relevancia en el contexto actual de dependencia agroalimentaria, colonialidad y etnocentrismo (Segato, 2014). Entendemos que dicho contexto ha motivado una explotación excesiva de los recursos naturales, un aumento acelerado del control sobre los alimentos, la energía y el agua, sumado a un incremento de la contaminación atmosférica, de los cuerpos de agua (lagos, ríos y mares), mayor contaminación de suelos, erosión y deforestación. Se rompen ecosistemas disminuyendo la diversidad biológica, atentando contra la propia soberanía a la hora de tomar decisiones sobre lo consumido y lo desechado.

Se nos hace urgente entonces volver sobre el trabajo artesanal y la conexión con la tierra, apelando una vez más a la soberanía alimentaria, la no dependencia de los mercados, y la conciencia sobre los alimentos que consumimos en una reflexión constante sobre este quehacer. El conocimiento sobre la producción del alimento, habilita una resistencia pedagógica en múltiples aspectos, abordando los problemas que acaecen en nuestros mundos de vida y permitiendo un proceso de prosumo (producción y consumo) no alienado de saberes.

La ecopedagogía y ecología de saberes como perspectivas, respecto a la institucionalización, las encontramos oportunas en el acercamiento entre la academia y la vida de las personas desde la posibilidad de conectar con la resolución de problemas primarios para la supervivencia y dignidad humanas. En 
el caso de las personas PLA, se incentiva salir al "aire libre", invitándoles y recibiendo su visita en el CeRP, cuestionando el aulacentrismo y la cotidianeidad instituída. De esta manera el aprendizaje desde una pedagogía praxística habilita la conexión entre la vivencia y la reflexión.

No fueron hallados antecedentes en relación al tema formación docente y contextos de encierro. Encontramos algunas referencias en una tesis de grado de la Facultad de Psicología de la Universidad de la República titulada "Contexto de encierro y educación: obstáculos y facilitadores desde una experiencia de asesoría pedagógica" de la Psic. Camila Pérez Martín (2016). El mismo aborda los vínculos entre docentes universitarios y estudiantes PLA desde un proyecto extensionista. Se identifica también un parcial presentado por estudiantes del IPA (Fernández y Goicoechea, 2011) del curso Investigación Educativa y parciales entregados en los cursos anteriormente mencionados del CeRP del Sur.

Consideramos que la experiencia concreta y reflexiva que llevamos adelante, así como este primer acercamiento en el camino hacia su divulgación, es un aporte original a la comunidad académica.

Tomamos como punto de partida los siguientes objetivos:

a) de la acción extensionista

$>$ Llevar a cabo una tarea que integre a diferentes comunidades educativas en un sentido expresivo, productivo y reflexivo.

$>$ Fomentar que los actores sociales participen junto a los centros educativos tanto en la planificación y la ejecución como en la evaluación del proceso.

$>$ Generar un proceso a través del cual se busca resolver problemáticas de la sociedad considerando los espacios y los tiempos de los actores sociales involucrados.

> Integrar nuevos ámbitos con la función de una mejor nutrición del proceso de aprendizaje, así como de un fortalecimiento de los vínculos entre distintos sectores humanos.

b) de los aprendizajes curriculares

$>$ Fomentar un proceso educativo transformador donde todas las personas involucradas aprenden y enseñan.

> Buscar un intercambio horizontal entre el saber académico con el popular.

$>$ Generar procesos de comunicación dialógica (procesos de comunicación y educación en los que no exista un educador, que deposite el conocimiento en un educando pasivo, sino en los que todos seamos educandos-educadores).

$>$ Coordinar desde la horizontalidad de los vínculos con todos los participantes, para que el proyecto se vivencie como una actividad intergeneracional, trascendiendo estereotipia de roles entre docentes y estudiantes.

$>$ Crear un modo de relacionamiento con el medio el cual permita descubrir 
nuevas áreas donde investigar, producir nuevo conocimiento y utilizar una metodología de aprendizaje integral y humanizadora.

\section{Área de estudio}

El espacio territorial donde parte la iniciativa y se encuentran las personas involucradas, estudiantes y docente del CeRP del Sur y personas PLA, es en el Centro educativo que forma a profesores de educación media, en Atlántida Norte. La categoría analítica en que basamos la mirada sobre los espacios institucionales y su dinámica e intercambio es la cartografía pedagógica (Ramírez, 2017). La misma propone abordar desde un dispositivo analítico la posibilidad de "develar de forma cualitativa intencionalidades, modos de organización, códigos, discursividades, acciones, bases teóricas, vínculos, creaciones, objetos y todos aquellos insumos que caracterizan el acontecimiento formativo entre sujetos" (Ramírez, 2017).

La Institución de formación docente para la enseñanza media CeRP del Sur, perteneciente a ANEP/CODICEN, se caracteriza por ubicarse en un contexto territorial suburbano. Se presenta como la única propuesta de formación terciaria del entorno. Sus estudiantes pertenecen a contextos urbanos, suburbanos y rurales situados en el interior del país. Ofrece becas de alojamiento, transporte y alimentación que habilitan a que la población estudiantil provenga de entornos socioeconómicamente vulnerados.

Como se ha mencionado, el tejido del vínculo con las personas PLA surge en el CeRP del Sur, en instancias de la presentación de la obra de teatro "El día después", escrita, dirigida y protagonizada por las mismas. Comenzó en ese momento la coordinación con la Unidad 6 "Punta de Rieles" del INR. En el desarrollo del proyecto incluimos el trabajo con "El Almendro Centro Cultural", ubicado dentro de la Unidad 4 "Santiago Vázquez" del INR (ex COMCAR), el cual presentaba una realidad considerablemente diferente por su autonomía del Estado. Con la Unidad 6 coordinamos en forma directa con los compañeros PLA, en cambio con la Unidad 4 la gestión era realizada por intermedio de la coordinadora del Centro Cultural, Rocío Morales, quien gestionó y sostuvo dicho centro.

La ejecución del proyecto transcurrió durante los años 2017 y 2018. Cabe destacar la distancia geográfica entre estas tres instituciones y por lo tanto el recorrido que reiteradas veces debimos transitar estudiantes y docente del Cerp del Sur o las personas PLA para llegar al encuentro. El Cerp del Sur se encuentra a la altura del Km. 45 de la ruta interbalnearia "Liber Seregni" y en el Km. 2 de la ruta 11 "Eladio Dieste". La Unidad $N^{\circ} 6$ a la altura del Km. 17,500 de la ruta 8, a una distancia de 40,2 $\mathrm{km}$ de la anterior. La Unidad $\mathrm{N}^{\circ}$ 4, a la altura del $\mathrm{Km} .20$ de la ruta 1 , a una distancia de $65 \mathrm{~km}$ y más de una hora de viaje en auto desde Atlántida. Los caminos no son directos y en más de una oportunidad perdemos el rumbo, tomamos rutas equivocadas, se nos dificulta llegar. Las vías de acceso son de tierra y lejanas a las rutas principales. Hay muy poca frecuencia de ómnibus. Entendemos que esta condición evidencia el carácter de "gueto" o periferia oculta de estas instituciones (Wacqüant, 1999). 
Nacen en el intercambio ideas que alentaron a pensar un trabajo conjunto, coparticipativo, limitado por la distancia, los límites del encierro y los destiempos de las personas involucradas.

El primer encuentro, en el Cerp del Sur fue de gran importancia porque sembró la posibilidad de cierta porosidad interinstitucional y de la extensión del profesorado. Por otro lado, observamos como punto de partida las complejidades y dificultades para acceder e intercambiar entre las personas que forman parte de estos territorios específicos, que se abordarán en los siguientes apartados.

\section{Material y Métodos}

La extensión crítica, pensada en clave de aprendizaje y acción en la formación del profesorado, no se encuentra institucionalizada desde el Consejo de Formación en Educación (CFE), jerárquicamente subordinado a la Administración Nacional de Educación Pública (ANEP/CODICEN). Este aspecto provoca la necesidad instituyente de co-crear entre estudiantes y docente del CeRP del Sur un espacio de trabajo que implique e incorpore desde la reflexión y acción todos los cursos que el estudiantado entienda pertinentes referentes a su formación específica, futuro perfil de egreso y rol profesional del profesorado, sumado a los cursos curriculares llevados adelante por la docente del área pedagógica, como nos plantea la extensión crítica:

Cómo se movilizan, cómo se inquietan, cómo pueden traducir las energías de juventud en cambios profundos de una institución que paulatinamente se ha ido sedimentando y, más allá de su carácter público conquistado por el movimiento estudiantil, aún hoy es de privilegiado acceso. (Medina y Tommasino, 2018, p.13)

Carbonell (2015) se manifiesta en referencia a la importancia de rescatar en clave pedagógica los antecedentes más emblemáticos de la formación, con el objetivo de mostrar que todo devenir educativo tiene su propia genealogía y nunca se parte de cero. El proyecto de extensión de formación del profesorado nace en el encuentro de la obra de teatro de Usina Cultural Matices presentada en el CeRP del Sur en el año 2017 y el Proyecto de huerta, bioconstrucción y expresión permacultural en el marco del curso de Pedagogía 1 desde el año 2015, interdisciplinario y transgeneracional. La primera actividad señalada surge en forma de acción artística. El objetivo era mostrar y compartir la realidad y el sentir de las personas privadas de libertad en primera persona y el proyecto mencionado, que desde la academia buscaba vincular los saberes populares relacionados al trabajo con la tierra con los saberes de la pedagogía en formación en educación. Surgió del encuentro la posibilidad de cruzar los saberes de las personas PLA, con los saberes científicos y populares que cada persona trae consigo y crear un proceso en común: un proyecto de extensión que permitiera generar vínculos entre los estudiantes de profesorado y las personas PLA.

A modo de estimación, las personas involucradas oscilaron entre unos diez estudiantes que fueron partícipes directos del proyecto y estudiantes que se relacionaron de manera esporádica en algunas actividades que alcanzan más de cien. De la misma manera, una docente estuvo involucrada a lo largo de todo el 
proyecto y algunos docentes apoyaron en momentos puntuales. Respecto a las personas PLA que participaron de la experiencia unas diez acompañaron a lo largo de todo el proceso y alrededor de sesenta participaron en talleres específicos.

Como grupo nos convocó la posibilidad de generar espacios de encuentro horizontal en relación a los saberes que se ponen en juego en los procesos de enseñanza y aprendizaje. En el transcurso pudimos asirnos de numerosas maneras de acción por y para el aprendizaje y la creación de nuevos conocimientos, aportando para poder reforzar el rol profesional del profesorado de cara a su futuro trabajo con estudiantes PLA.

En retrospectiva, al reflexionar sobre el trabajo metodológico, entendemos que el mismo va de la mano con cierta espontaneidad didáctica donde prima el diálogo entre todas las personas involucradas. Se presenta desde el comienzo una búsqueda explícita por no seguir un método instrumental, sino construir experiencia del orden del saber teniendo presente el diálogo entre la intersubjetividad y el acontecimiento didáctico (Litwin, 1997; Chevallard, 1998). Coordinamos cada espacio y tiempo en conjunto para luego pensar los materiales e insumos. Planificamos las actividades, dependiendo de quiénes llevaban adelante el taller (las personas del CeRP, las personas PLA o en conjunto).

Litwin (1997) expresa como elemento primordial del método de las configuraciones didácticas la comprensión de los contenidos, en tanto favorece los procesos reflexivos como motor de la producción de conocimientos. En este sentido, sistematizar la experiencia ha generado un espacio de movimiento interinstitucional (CeRP-Unidades del INR), intergeneracional (múltiples franjas etarias y generacionales del mundo adulto) e interdisciplinario (saberes de los estudiantes PLA y del estudiantado del CeRP de las diferentes carreras del profesorado).

Así es que los procesos de enseñanza y aprendizaje tienen la posibilidad de situarnos en un diálogo. Como suceso acorde a estos se prima la diversidad, dando cabida a prácticas de largo recorrido.

En cada oportunidad que regresamos de los encuentros, elaboramos una hoja de ruta donde plasmamos en forma exhaustiva y grupal (estudiantes y docente de pedagogía de formación del CeRP del Sur) las impresiones respecto a las jornadas de trabajo. El conjunto de hojas de ruta impresiona una bitácora, donde las experiencias quedaron plasmadas desde nuestra perspectiva. Fuimos ordenando coordenadas para el reconocimiento de las mismas, adjudicándose referencias respecto a la Unidad donde fue la actividad (UN4 o UN6) y el mes y año de cada una (mes y año abreviados).

Como mencionamos anteriormente, a lo largo de los años 2017 y 2018 nos fuimos involucrando y compartiendo actividades cotidianas en los respectivos espacios institucionales (CeRP del Sur - Unidades del INR), para poder habilitar el "ida y vuelta". A modo de ejemplo, durante el desarrollo del proyecto, un grupo de estudiantes PLA nos invitó a participar de las VI Jornadas de Extensión del Mercosur donde nos encontramos con Tommasino. En diálogo con éí, conocimos una postura metodológica que se asemejaba de manera considerable con nuestro trabajo: la denominada Extensión Crítica (Medina y Tommasino, 2018). 
A su vez, surgió desde el espacio áulico formal y en el diálogo con las personas PLA la necesidad de abordar una pedagogía permacultural, teórico-vivencial, donde se incluye el acercamiento hacia la autonomía alimentaria, el prosumo, la bioconstrucción y la mirada en clave de género.

Entendemos que esta forma de interpretar el saber, trabajada en el curso de Pedagogía 1 y Observación y Análisis de las Instituciones Educativas del CeRP del Sur, se inscribe en una mirada ecoepistemológica:

Es una ecología porque está basado en el reconocimiento de la pluralidad de conocimientos heterogéneos (uno de ellos es la ciencia moderna) y en las interconexiones continuas y dinámicas entre ellos sin comprometer su autonomía. (de Souza, 2010, p.49)

Desde el punto de vista de Jaume Carbonell (2015), la transdisciplinariedad es una categoría que convoca a intervenir de diferentes maneras hacia un objetivo crítico. Las posibilidades no se agotan en lo que ya está descrito. Problematizamos la relación vertical e iluminista que proponen algunas formas hegemónicas de presentarse frente a los grupos o colectivos sujetos de la extensión, lo cual es legitimado históricamente. El pedagogo uruguayo Julio Castro (2007) plantea en este sentido que "la enseñanza dictada por el maestro mediante un dogmatismo que no admitía críticas, imponía, por último, al niño el rol de un recipiente pasivo y silencioso. El maestro trataba de llenarlo, como si fuera un odre, con su sabiduría mágica, más allá de toda crítica y de toda demostración" (Castro, 2007 pp. 85-86).

Construir el método implica entonces, en este momento concreto, revisitar y sistematizar parte de la experiencia y sus desafíos.

\section{Discusión: desafíos de la formación y práctica profesional docente: ecocrítica y resistencia pedagógica}

Entendemos como un primer desafío que la extensión se institucionalice en Formación Docente, "integrada al quehacer" de la cotidianeidad de la formación del profesorado, superando lo que realmente es, un "gueto del compromiso social" ajeno a "nuestra propia comunidad académica" (Medina y Tommasino, 2018, p.9). Si no están institucionalizadas, las iniciativas comunitarias/extensionistas deben necesariamente partir de los estudiantes y docentes que traen consigo el impulso del compromiso social, generando un esfuerzo excepcional muy grande que por un lado va en desmedro de la acreditación para la titulación y por otro implica un esfuerzo económico y de tiempo personal que se dificulta sostener. En otro sentido, integrar al quehacer cotidiano las iniciativas comunitarias/extensionistas supera el aislamiento académico y promueve una nueva cultura institucional de la formación del profesorado de cara a los problemas específicos del medio donde se sitúa.

Se percibe a grandes rasgos, que la formación docente no requiere de extensión porque en su labor profesional y en el curso de la carrera lleva implícito el contacto con la comunidad a través de la enseñanza en las aulas, pero observamos que dicho contacto no es suficiente para la formación.

La formación docente se ha desarrollado fundamentalmente a nivel de cursos de grado, estrechamente vinculada al campo de la 
inserción laboral, prioritariamente vinculada a la enseñanza primaria y media. Es de destacar, además, que ha estado esencialmente centrada en cuestiones de enseñanza si pretendemos compararla con los fines de la formación universitaria. (Salvá, 2011, p.11)

El trabajo nos permitió "sumergirnos en la posibilidad de enseñar y aprender" buscando fracturar "los estereotipos de educador y educando, dando lugar a una terceridad que interpele las jerarquías de esta relación diádica y amurallada entre docente y alumno" (Medina y Tommasino, 2018, p.10), potenciando los vínculos con la comunidad.

Compartimos que "hay una cuestión transversal (...), el rol del estudiante, cómo asumen el protagonismo en esta coyuntura" (Medina y Tommasino, 2018, p.13). Entendimos clave que también las personas PLA pudieran involucrarse en el proyecto y en su aspecto instituyente desde el protagonismo y la cocreación, dado que también serían los estudiantes de los docentes egresados de la formación del profesorado. En este sentido, se pensó la acción extensionista desde la horizontalidad de las propuestas y vínculos, aportando cada persona involucrada desde su espacio de saber, tanto de la experiencia, como del orden de la sensibilidad, el trabajo manual y el intelectual. Los mundos de vida se entretejieron, revalorizando la riqueza del encuentro y el trabajo.

Estamos en constante cuestionamiento de nuestros roles, presentándose un segundo desafío: la construcción de sistematicidad de la acción extensionista para evitar caer en una imposición cultural. La sistematización de la experiencia extensionista que nos convoca en esta instancia se motiva desde la conciencia de la importancia instituyente que presenta el trabajo, para que no quede en mero voluntariado asistencialista. Pretendemos dar respuestas a los problemas propios de nuestro tiempo y espacio, específicamente latinoamericano, y de los sujetos educativos institucionalizados involucrados en el proceso.

Comprendiendo a las personas PLA como sujetos del derecho a la educación, observamos que el mismo les ha sido privado e invisibilizado por la sistemática criminalización de la pobreza. Wacqüant (1999) en su obra "Las cárceles de la miseria", en el prefacio de la edición para América Latina, explicita que las políticas de seguridad de la última década del siglo XX, en EE.UU. y Latinoamérica se vincularon con la noción de "limpieza de clase". Se describe discriminación y represión selectiva contra personas que pertenecen a ciertos grupos sociales y/o etnoraciales en ciertas zonas simbólicas. Luego, en su trabajo explica cómo el Estado de bienestar en el capitalismo neoliberal contrajo dicha "limpieza", para dar paso a un Estado mínimo represor de las clases subalternas:

[L]a excesiva generosidad de las políticas de ayuda a los indigentes sería responsable del ascenso de la pobreza en los Estados Unidos: recompensa la inactividad e induce la degeneración moral de las clases populares, y en especial esas uniones "ilegítimas" que son la causa última de todos los males de las sociedades modernas, entre ellos las "violencias urbanas". (Wacqüant, 1999, p.26)

Se legitima una guerra sin cuartel contra los pobres y marginales del nuevo orden económico, avanzando bajo la insignia de la recuperación de la "libertad 
perdida".

Observamos una paradoja: unos buscan recuperar la libertad encerrando a los otros, a través de la legitimación de la lógica de la intolerancia selectiva y la naturalización del aislamiento social. El ambiente pedagógico que legitima el ideal de aislamiento surge en el siglo XIX. En este sentido Julio Castro (2007) planteaba que el proceso de represión comenzó a consolidarse en el advenimiento del higienismo decimonónico y la capacidad de ordenar en un estadio de conocimientos a las clases sociales dentro de una tecnología pedagógica donde el estudiante estuviera aislado, quieto y en silencio:

Pero como además, la enseñanza se reducía a oír y contestar, en medio de actividades exclusivamente intelectualistas, hubo necesidad de buscar el ambiente propicio para lograr, lo más ordenadamente posible, esa quietud. Por otra parte, una de las características de la escuela tradicional fue su cerrado individualismo: ni trabajos en grupos, ni realizaciones que no surgieron del esfuerzo propio y personal. Esto tuvo a su vez, como consecuencia, el ideal del aislamiento. El niño además de estar callado y quieto, debía estar solo. (Castro, 2007, p.85)

A partir de esta naturalización del educando quieto y solo, se genera un concepto de ilegitimidad de otros formatos pedagógicos y propuestas de enseñanza y aprendizaje:

Toda realización mutua se consideró o una cooperación ilícita, o una fuente de perturbación y anarquía. Los niños-40 o 50 en una clase-debían estar solos, cada cual por su lado. (...) Un individualismo exacerbado presidía el espíritu de la escuela. Como el hombre realizaba en el exterior la lucha por la vida, el niño, en la escuela, vivía también en un clima de lucha por su superación, frente a los demás. (Castro, 2007, p.85-86)

A partir de esta forma de concebir la enseñanza, desde el control total y la mirada constante vigilante de la autoridad, la arquitectura de las instituciones actúan en pro de ello. De esta forma es identificada dicha tecnología pedagógica como panóptico por las personas PLA. La estructura arquitectónica y pedagógica del panóptico se define como una torre de vigilancia central con un anillo de celdas separadas entre ellas; desde la torre se ve todo, pero desde las celdas no pueden verse entre sí. El panoptismo "debe ser comprendido como un modelo generalizable de funcionamiento; una manera de definir las relaciones del poder con la vida cotidiana de los hombres" (Foucault, 2002, p.189). Es un modelo que nos permite entender lo que denomina la sociedad disciplinaria. Se garantiza a través de la disciplina del cuerpo la funcionalidad al orden imperante en forma de utilidad y obediencia:

La disciplina aumenta las fuerzas del cuerpo (en términos económicos de utilidad) y disminuye esas mismas fuerzas (en términos políticos de obediencia). En una palabra: disocia el poder del cuerpo; de una parte, hace de este poder una "aptitud", una "capacidad" que trata de aumentar, y cambia por otra parte la energía, la potencia que de ello podría resultar, y la convierte en una relación de sujeción estricta. 
(Foucault, 2002, pp.126-127)

Genera además un vínculo de retroalimentación entre estos dos elementos, en un mecanismo que "lo hace tanto más obediente cuanto más útil, y al revés" (Foucault, 2002, p.126). Es una utilidad que potencia la sumisión obediente del cuerpo que sujeta la conciencia y limita las posibilidades de creatividad, expresión y prospectiva.

En esta disociación se deshumaniza a la persona. Entendiendo el ser humano como ser biopsicosocial, se puede ver un desmantelamiento de ese carácter en la imposibilidad del reconocimiento con otro ser humano. Se acentúa la soledad y la falta de vinculación, generando un ambiente que contribuye a una atomización de las relaciones sociales. Las potencialidades son cercenadas en función de la utilidad; la creatividad y la expresión, todo aquello que no es dar vuelta a la rueda del capital, queda excluido de las posibilidades de ser desarrollado. Cada quien frente a su tarea, al lugar que la sociedad le depara, y en soledad:

La multitud, masa compacta, lugar de intercambios múltiples, individualidades que se funden, efecto colectivo, se anula en beneficio de una colección de individualidades separadas. Desde el punto de vista del guardián está reemplazada por una multiplicidad enumerable y controlada; desde el punto de vista de los detenidos, por una soledad secuestrada y observada. (Foucault, 2002, p.185)

Uno de nuestros objetivos es reconsiderar y reformular dichas lógicas para una educación por y para la emancipación.

Un último desafío que identificamos se relaciona con "disputar el 'espacio académico central' que es el acto educativo (áulico)" (Medina y Tommasino, 2018, p.10). Entendemos, como plantean Medina y Tommasino, "fundamental generalizar las prácticas integrales en los estudiantes para la convivencia natural de la investigación y extensión del cursado, también en la incorporación desde un vínculo dialógico-crítico con actores sociales organizados y en procesos de luchas" (Medina y Tommasino, 2018, p.10).

Estos espacios (extensión en formación docente y contextos de encierro), de perfil comunitario se construyen muchas veces desde la superación del conflicto, recorrido que alimenta la reflexión y acción sostenidas, la resistencia cultural y el cuestionamiento del status quo que incomoda. Esa incomodidad genera reacciones diversas, que luego se busca resignificar.

Como anteriormente planteamos la resistencia nace aplicando lo que de Souza (2010) denomina ecología de saberes. Implica una heterogeneidad no sólo de saberes sino de epistemologías y formas de concebir el mundo diversas, incluyendo la ciencia moderna pero no únicamente ni por sobre las otras, sino como una forma más de acceso al conocimiento a la par de lo que puede ser la permacultura o los saberes ancestrales. De esta forma se construye según el autor una epistemología del sur.

Asimismo, la ecología de saberes entiende la ignorancia como una valoración de conocimientos donde lo que se aprende vale más que lo que se sabe. 
Se aspira a la utopía pedagógica donde aprender nuevos conocimientos no implique olvidar ni desmerecer el propio (de Souza, 2010). Problematizamos el concepto, entendiendo que la noción de futuro posible se acerca desde un pensamiento latinoamericano a nuestro enfoque: la idea es ir trazando el camino, y no necesariamente sus objetivos son utópicos.

La importancia de una ecología de saberes radica en que todos los conocimientos son incompletos y ninguna forma de conocimiento puede dar respuesta a todas las intervenciones y formas de existencia. La copresencia de saberes habilita la posibilidad de dar respuesta a más aspectos y de forma diversa. Al mismo tiempo, cuando ante una intervención en el mundo real se pueda aplicar más de un conocimiento, el autor señala que se debe priorizar "aquel que garantice el mayor nivel de participación a los grupos sociales involucrados en su diseño, ejecución y control" (de Souza, 2010, p.56).

La multiplicidad de construcciones praxísticas y el alto nivel de participación son posibles desde la pedagogía de la mesa colectiva: "Luzuriaga, al establecer un criterio sobre qué es nueva educación, la sintetiza en cinco principios muy generales que, a la vez caracterizan el movimiento pedagógico moderno, y expresan las ideas actuales sobre los niños. Esos principios son: Vitalidad, Actividad, Libertad, Infantilidad, Comunidad" (Castro, 1941, p.105). Ambiente y territorio humanizado se conjugan en el encuentro pedagógico de la mesa colectiva, poniendo sobre dicha mesa todo aquello que construye un saber vivo, activo, libre y comunitario en constante movimiento.

Apelar a pedagogías que generen impacto en la experiencia de las personas, es base de lineamientos pedagógicos que se entrelazan y concluyen en nuevas formas de significar los conocimientos. Desde una perspectiva montessoriana, repensar el aprendizaje social y el ambiente pedagógico se transforman en factores fundamentales a la hora de la internalización del conocimiento (Carbonell, 2015).

El anterior campo de conocimiento pedagógico se apoya en teorías que nos sugieren nuevas posibilidades de construcción del rol profesional docente, de cara a un encuentro desde la formación y la acción extensionista con nuestros futuros estudiantes PLA.

\section{Resultados}

La pertinencia de proponer un proyecto con la comunidad educativa, y específicamente en formación docente, que permita concientizar y habilitar las decisiones sobre los procesos productivos se justifica en el desconocimiento práctico de cómo obtener los recursos. En este sentido, los elementos que proponernos y el hecho de concretar la construcción de una cocina "Rocket", compartir la huerta, observar la construcción de bancos con bidones, etc. implican un ensayo, la demostración de una posibilidad, la transformación embrionaria, y por ende, plausible. Esto se da en hechos concretos, en adquisición de habilidades y conocimientos, pero también en un horizonte epistemológico hacia la visualización de la reutilización y la permacultura en general como una alternativa pedagógica:

Todos nos fuimos con más saberes. Entendemos que es 
importante concientizar cuando se generan estas formas de conocimiento, que son distintas a las que pregona la ciencia positivista, la matemática, la estadística. Los que estuvimos ahí sabemos que el conocimiento que adquirimos ese día es sumamente valioso. Es conocimiento directo, construido emancipadamente a través del arte. (Un4/feb18)

Nos convoca la reflexión sobre la acción misma y no encontramos un resultado concreto, plenamente identificable, sino un proceso cuyo comienzo es posible reconocer, pero no así su final. Concretamente, no es posible cuantificar resultados de esta experiencia, o medir los alcances en personas influidas por la misma. Es así que entendemos como resultado la observación reflexiva de lo acontecido en el trabajo ofrecido y recibido, comprendiendo este proceso dialógico como un ir y venir transformador. En este sentido, el resultado se amalgama con la noción de acción extensionista.

El trabajo concreto de encuentro y propuesta de construcción de una cocina de barro denominada "Rocket" se consolida en la Unidad 6. Fue inspirado por el proyecto de Huerta del CeRP del Sur, contenidos específicos del campo pedagógico y la Huerta orgánica "Tupinambá" que tuvimos el gusto de conocer por primera vez en una salida didáctica de formación en la misma Unidad del INR. El diálogo entre todas las personas involucradas se dio sin dificultad. Se intercambiaron libros y promesas de regreso, gestando la posibilidad de la construcción en clave de ecología de saberes. Se habilita así la construcción de una mesa colectiva (Castro, 2007).

De esta manera se cuestiona la criminalización de la pobreza, se desdibuja el panóptico y demostramos una adaptación a la forma de trabajo que no está alineada con esas políticas institucionalizadas. Las reacciones ante dichas políticas suelen acarrear violencias. El ambiente se hostiliza. Lo vivenciamos en una de nuestras visitas:

Esta mañana invernal se da en contexto de un reciente motín en el módulo 12 del ex COMCAR que nace como consecuencia, entre otras cosas, de las condiciones infrahumanas y denigrantes que aún gobiernan en gran parte del sistema penitenciario uruguayo. Este lunes gris y frío también se da en un marco de ocupaciones que se extienden por una comunidad educativa harta de ver sus necesidades postergadas y menospreciadas. (Un4/jul18)

La organización que se construye tanto para amotinarse como para trabajar, tal vez hace de la vida y los ambientes que habitan las personas PLA un todo colectivo que a pesar de las circunstancias, muestran el lazo social un poco más fortalecido.

El trabajo colectivo horizontal implica que no existan calificaciones, más que del propio colectivo hacia su trabajo, posibilitando su reformulación constante. El saber cómo bien común es una idea que disputa el espacio central de la creación de conocimiento. La producción colectiva del saber nos enriquece desde la participación, apropiándonos de la misma. Construimos un ambiente humanizado fortaleciendo lo individual y lo colectivo en un hacer no competitivo. Nos acercamos a una creación intersubjetiva reflexiva y práctica: 
Desde nuestro punto de vista repleto de intersubjetividad pudimos ver de cerca que nuestra humanidad supera individualidades, que podemos hablar con nuestra expresividad visual, que podemos salir del lugar de víctima y podemos cuidar de nuestro "yo niño" y ser nuestros propios padres, hermanos, amigos. Cuanto más próximos estemos de ese pequeño niño que fuimos más fuertes seremos hoy.(Un4/feb18)

El día que concretamos la planificación de la construcción de la cocina "Rocket", fuimos a compartir un espacio de performance que denominamos "Mirarte" (Un6/nov17). La misma consistió en intervenir el espacio desde la expresión corporal apelando a una comunicación no mediada por la palabra. La acción constructiva colectiva asiste a un estado pleno de creación y conciencia general promovido por dicho trabajo, enfrentando la alienación de la corporeidad institucionalizada, en el proceso de concientización y generación de herramientas para la decisión de los procesos productivos. En este marco surge la idea desde las personas PLA de que "la cárcel es como muchos panópticos, todos te miran, todos saben lo que haces, donde estas, pero nadie se ve realmente" (Un6/nov17). Se parte de la mirada performática, sentados frente a frente. Utilizando el lenguaje corporal y gestual se reconocen los rostros, los ojos, la expresión. En el encuentro con las otras miradas se comienza a desarticular el ambiente de vigilancia.

Surgen múltiples miradas validadas por las del colectivo, las cuales pueden inhibir la pertinencia del panóptico. La vigilancia deja de ser la única mirada legítima. Nos vemos con detenimiento, con dedicación, con importancia real, tratando de interpretar sin que nadie sepa el contenido de nuestra comunicación. Por otra parte, ante la experiencia horizontal, descubrimos que sostener la mirada parece una actividad simple, pero que no es tan fácil generar una comunicación sin verbalizar. También se pone en evidencia la hiperverbalización que llena espacios gratuitamente, y se revaloriza el silencio.

Retomando el análisis del trabajo en la cocina "Rocket" en Punta de Rieles, observamos que a partir de la construcción con las manos, surgió la idea de lo que es pedagógicamente posible. Tal vez sea de las primeras veces que en un centro de privación de libertad, en medio del cemento recalcitrante, se construye con barro arcilloso, arena, agua, fibra que fue poda recuperada para su bioutilización y herramientas de construcción que se obtuvieron del propio entorno, por los internos, por estudiantes, por la docente, por actores sociales vivos. Al cemento lo interpretamos como representación de los estilos de construcción hegemónicos, legitimados, con afán civilizatorio. Material que impresiona sensiblemente desde la frialdad, en contraposición con la calidez térmica del barro.

La falsa dicotomía manual intelectual, a través de la alienación, permite interpretar un concepto de trabajo como individual, cuando en la práctica no experimentamos otro trabajo que no sea colectivo: "Reafirmamos la importancia de la construcción reflexiva y humana a partir del trabajo manual. Somos a partir del trabajo. Creamos y crecemos a partir del trabajo. Y qué grato que la tarea se disfrute y se realice en compañía" (Un6/dic17). La visualización del trabajo como colectivo sólo es posible a través del trabajo no alienado, es decir de la reflexión y acción mediadas dialécticamente por los aprendizajes con sentido. El trabajo no 
alienado implica no sólo la dimensión reflexiva, sino también la material, al hacer usufructo del producto de nuestro trabajo. Todo esto se cumple en el proceso de la cocina "Rocket', rescatando particularmente su legado respecto a la utilización de los espacios colectivos de las personas PLA luego de finalizado el proyecto, cuyo registro observamos en redes sociales.

Surge de una entrevista realizada a una de las personas PLA la noción de "buen sentido de comunicación" (Un6/dic17). Entendemos esta reflexión como la internalización que para trabajar colectivamente es condición necesaria crear espacios de diálogo y reconocimiento. El tema se venía conversando en jornadas anteriores. El trabajo manual colectivo implica necesariamente un trabajo intelectual que se expresa en la comunicación horizontal. De lo contrario sería reflexión, trabajo y vínculo alienado. El concepto "descarte" aparece en las reflexiones de las personas PLA referidas a la revalorización de elementos que son desechados, así como observamos los procesos de reconocimiento humanizante de las personas que también son "descartadas" socialmente.

El cuerpo posteriormente institucionalizado para ser reprimido, se transforma a partir de experiencias compartidas de trabajo no alienado en cuerpo vivo. Una persona PLA referente del Almendro en talleres relacionados con arte manual llegó al encuentro, y nos compartió

sus ideas, motivaciones y visiones sobre el nuevo taller de artesanía que llevará adelante a partir del segundo semestre del presente año. (...) tiene el don de resignificar lo real con sus manos llenas de magia, con su imaginación sin cadenas y con sus palabras honestas, está convencido de la importancia de ampliar el alcance de espacios educativos como los de este centro cultural. (Un4/jul18)

Asimismo, en este encuentro, dicho referente coordinó con otros dos talleristas, estudiantes del CeRP, de las carreras de Filosofía y Matemática respectivamente, $\mathrm{y}$ en conjunto tomaron

las riendas y comienzan mostrando los diferentes tipos de hilos, semillas, herramientas y técnicas. Despliegan teóricamente, utilizando un powerpoint, la historia del macramé, reflexionando sobre el valor de rescatar los saberes originarios y comunitarios. Al cabo de unos minutos ya estamos todos compenetrados con nuestras tablitas creando y transformando nudo a nudo. Quienes sacan la movida más rápido tiran una mano a quienes nos lleva un poco más de tiempo. Todos tenemos el cuerpo involucrado en el hacer transformador y el corazón involucrado en el diálogo con el de al lado. La adhesión y recepción es total. En conversaciones posteriores al taller (...) se mencionaba la importancia de traducir las intenciones en acciones prácticas y sobre lo gratificante que ello resulta. (Un4/jul18)

Retomamos la idea de la construcción participativa del ambiente de aprendizaje, co-creación de los saberes como bienes comunes y la importancia del disfrute en los procesos de enseñanza que articula lo manual e intelectual en forma creativa, sin estereotipos ni coacción. 
En relación al valor epistemológico de la bioconstrucción y la ecopedagogía, reflexionamos sobre lo significativo del "aprender a hacer una cocina con cero costos y en forma permacultural, aprovechando los elementos que desde otras lógicas son de 'descarte', la felicidad de los que metían las patas el el barro, el descubrir que, así como se hace una cocina, o un horno, se puede hacer una casa y que esas cosas existen de verdad" (Un6/dic17).

Identificamos, por ende, nuestro campo de conocimiento como pedagogía crítica. Se fundamenta en la búsqueda de la toma de decisiones sobre cómo nutrirnos, la soberanía alimentaria y de saberes y la siembra consciente. Por esta razón surge en el diálogo la necesidad de una cocina donde preparar los alimentos. La actividad autónoma de cocinar antes de construir la cocina "Rocket" no era de fácil acceso en el centro penitenciario:

Proyectamos para la próxima hacer una cocina "Rocket" a un costado del local de la cooperativa y en alguna otra oportunidad hacer una comida de olla entre todos. Esto surgió a raíz de que uno de los temas que surge cada vez que vamos es la alimentación. La mala calidad de la comida que les dan ahí, los altos costos de lo que les venden adentro y la dificultad para entrar cosas de afuera. (Un6/nov17)

El hecho de hacer pone de manifiesto los saberes previos, la actitud y aptitud hacia el trabajo y la memoria de los mismos como músculos que se reaniman, confiriendo sentido en la intervención de los espacios físicos. Un compañero PLA

amasó el barro con el pasto como si fuera un pan, alguien le preguntó si había trabajado en panadería y él dijo que sí. Hicimos referencia a la memoria corporal. Hacemos y repetimos inconscientemente los movimientos que tenemos incorporados. Todo es producto del aprendizaje. (Un6/dic17)

La elaboración de una descripción, interpretación y transformación del ambiente se vuelve base de las diferentes fuentes del saber. Los territorios subjetivos se construyen colectivamente en la revalorización de los aprendizajes, el rescate de los saberes y el trabajo, lo cual nos coloca en un encuentro de paradigmas pedagógicos y por tanto políticos, reivindicando la ecología de saberes.

En este sentido, aparece como elemento pedagógico concreto la autoproducción agrícola orgánica, uno de nuestros puntos de partida y motivación inicial. En muchas ocasiones los alimentos llevados a los espacios de encierro fueron de nuestras huertas, producto de nuestro trabajo transformador sobre la tierra. Así es como conocimos y nos acercamos a "Tupinambá", la huerta que las personas PLA trabajaban y cuidaban en la Un. 6, e intercambiamos sobre el método hidropónico desde la praxis de la pedagogía permacultural. Tras una jornada de cosecha compartida empezó la producción conjunta de comida en la cocina "Rocket". En diferentes días de encuentro cocinamos juntos guisos y pizzas. Asistimos nuevamente a la mesa colectiva:

Así se dio esta vez: sub grupos que estuvimos cómodos y que conversamos largamente. Almorzamos un rico guiso con verduras de su huerta y de las nuestras. Todos sentados en una gran mesa compartimos 
charla y alimentos. (...) Luego de la sobremesa sonaron tambores y bailamos candombe, hoy, en un sentido de resistencia. (Un6/mar18)

El taller de candombe había sido previamente acordado, y en esa instancia nos enseñaron a tocar los tambores. De esta manera nos encontramos con una reciprocidad pedagógica. Las personas PLA nos brindan un taller de percusión afrouruguaya: chico, repique y piano. En un intercambio horizontal, surge la ida y vuelta de saberes, desde el disfrute, la nutrición mutua, y el rescate reflexivo de la memoria colectiva.

Por otra parte, nos encontramos en contraste nuevamente con lo legitimado, preestablecido y no dicho: el castigo como construcción social de una penitencia carente de todo goce, que en el marco legal no es puesto de manifiesto, dado que las personas están privadas de libertad ambulatoria y sólo eso. "Fuimos contraste de realidad y humanidad. La expresión continuó con canciones. Llegó la música. No interesa la aptitud, si, la actitud de expresar y compartir tan hermosa actividad que cala nuestra cultura en nuestra piel" (Un6/mar18).

El hacer conjunto y los saberes compartidos nos invita a reflexionar sobre el encuentro de intereses y aspectos que alimentaron el sentido del grupo. En instancia de visita con varones del módulo 10 de la Unidad 4 del INR, estos reflexionan sobre el porqué de nuestra visita en relación a los vínculos, "para que no nos vean como unos pichis," decían, "y para que ustedes no nos vean como tal o cual cosa" respondimos (Un4/nov17).

En otra oportunidad, el colectivo de "El Almendro" y nuestro colectivo "Expresión y Diálogo" recibimos en la Iglesia de la Unidad 4, a las talleristas del Encuentro Latinoamericano de Teatro de las personas Oprimidas (ELTO) para contribuir a un cuerpo integrado a una emoción, lejos de la alienación. De esta manera la ecopedagogía y el encuentro con los saberes de nuestros territorios humanizados se asienta en una decolonialidad. Valoramos la actitud no hegemónica de las talleristas españolas, que en el marco del ELTO hicieron alusión a su situación de origen: "una de las talleristas que guió el taller al principio de todo cuando se presentó puso en claro su situación de española: pidió disculpas por la invasión de su pueblo en estas tierras" (Un4/feb18). Observamos una crítica geopolítica y pedagógica respecto al colonialismo eurocéntrico.

De esta manera, encontramos un paralelismo con la conciencia ante el necesario respeto y reconocimiento a la historia de los colectivos integrados por las personas PLA y los saberes y construcciones culturales previas a nuestra llegada. No pretendemos colonizar sus mundos de vida, sino por el contrario, reconstruir los mundos de vida de las instituciones y personas involucradas en el proceso de encuentro y aprendizaje.

A modo de cierre del análisis de la experiencia extensionista, rescatamos la reflexión de la hoja de ruta del día de encuentro/taller de alimentación y música:

Queremos hacer hincapié en la necesidad de un diálogo sincero que nos permita ser claros con nosotros y con el resto, afirmar nuestras diferencias, entenderlas, respetarlas $y$ con todo ello seguir construyendo. Agradecemos las diferencias que provocan tensiones 
porque son ellas las que nos permiten crecer. Que ellas aparezcan es un buen síntoma de que los lazos se están tejiendo, no permitamos que ello se convierta en un obstáculo que bifurque nuestros caminos, porque de esa forma estaríamos reproduciendo el modelo hegemónico que nos incita (y hasta qué punto en tantos aspectos de nuestra vida) a caminar solos. (Un6/mar18)

Como punto de unión entre este camino que proyecta y se proyecta en colectivo y de manera particular, se asienta una práctica que se hace coherente para comenzar dicha senda. El territorio humanizado se hace camino, como escribe el poeta, "Caminante no hay camino, se hace camino al andar" (Machado, 1912).

\section{Conclusiones}

Los desafíos presentados al comienzo del presente trabajo fueron abordados desde los objetivos planteados al comienzo de la acción extensionista. El territorio que sirvió como escenario de nuestro trabajo, se hizo familiar con la suma de encuentros en lo referente a las formas de las instituciones tanto a nivel material como orgánico.

El trabajo concreto desplegado en cada encuentro fue marcando distintas posibilidades en dicho territorio que apuntan a humanizar los espacios para las personas, y las personas hacia los espacios.

Por tales motivos podemos decir que hemos dejado huellas: en lo arquitectónico, una cocina "Rocket" presente y disponible para que las personas PLA puedan transformar su realidad cotidiana y que materializa nuestra intervención. En lo que refiere al CeRP del Sur como el otro espacio institucional, como primer experiencia de extensión se pudo colocar un precedente, así como la visualización de la falta de acuerdos de la formación del profesorado, lo cual dificulta el desarrollo de la extensión como parte de la educación en relación al perfil del egreso profesional. En este sentido vemos cómo esa construcción varía entre personas y grupos, siendo nuestro aporte el campo de conocimiento desde donde partimos, la experiencia sostenida durante dos años, y el trabajo de sistematización y divulgación del proceso.

En la expectativa sobre el rol del futuro docente se pauperiza la formación en relación a la educación de nuestros futuros estudiantes PLA, no teniendo en cuenta la especificidad de su situación de vida y las necesidades educativas integrales que demandan. Esto deriva en una realidad que observamos dentro del estudiantado de formación del profesorado donde se profundizan los prejuicios en la posibilidad del contexto de encierro como espacio laboral futuro.

En el CeRP del Sur recibimos en dos oportunidades a las personas PLA que presentaron sus obras de teatro y música. Dar lugar a estos encuentros fue de muy difícil andamiaje, pero dejó una serie de experiencias, sentimientos y vínculos que rompen prejuicios sobre las personas PLA y que constituyen un gran aporte a la formación, además de fortalecer las ideas de reciprocidad y horizontalidad. Esto contribuyó no sólo al intercambio grupal, sino también a toda la comunidad educativa que se vio interpelada con estos encuentros. 
Los elementos pedagógicos basados en la ecoepistemología, que se presentan en esta experiencia extensionista, son: el macramé, tejido en base a nudos de origen persa que es adoptado en nuestras regiones adecuándose a sus necesidades y estéticas, representa el trabajo no alienado, el artesanato como digna posibilidad de vida, respetuoso del entorno y diseñando a escala humana; el candombe, como clara demostración de resistencia anticapitalista del territorio afrouruguayo; la bioconstrucción en barro que nuestros ancestros en el campo ofrecieron desde el hogar y el rescate de saberes interculturales originarios; la expresión artística a través del aporte del ELTO, desde un taller de teatro de las personas oprimidas, cuyo origen se sitúa en Brasil de la mano de Boal, basado en la pedagogía del oprimido de Freire y, finalmente, la experiencia artística de performance desde la expresión corporal que denominamos "Mirarte", la cual nos enfrentó a nosotros mismos desde una comunicación interpelante de la mirada que vigila y que coloca el propio verbo en otro ángulo reflexivo y vivencial.

El ambiente vivo que se nos muestra habilitando la observación nos ofrece una perspectiva que, sumada a nuestras concepciones del mundo, posibilitan la construcción de saberes. Así los elementos desplegados a lo largo del trabajo en su conjunto permiten la transformación colectiva del territorio humanizado.

Las influencias tomaron direcciones múltiples: para quienes fuimos participantes directos, se hace evidente que un trabajo de dos años conforma una historia transformadora. En cuanto al resto de la comunidad educativa, a pesar de que el trabajo extensionista no contó con apoyo general y no logró calar como proyecto de centro institucional, se gestó un nuevo grupo de trabajo que hoy permanece en la misma línea con mujeres en situación de privación de libertad ambulatoria.

Hubo un encuentro de saberes que se promovió desde los relatos colectivos y la construcción de vínculos ecopedagógicos. La presentación del grupo de manera horizontal posibilita el intercambio de conocimientos sin prejuicios y se manifiesta en los quehaceres compartidos. Los conocimientos de huerta, y de la naturaleza en general, se visibilizan como un interés común desde donde partir en la construcción del orden del saber.

Concebir la horizontalidad tras décadas de educación piramidal, donde el docente es el dueño del saber y quien presenta las consignas tras un estudiantado pasivo que obedece, se hace muy difícil de implementar sin seguir esta línea pedagógica tradicional. Por ese motivo la interpretación de la horizontalidad fue muy difícil de transitar, generando desencuentros, los cuales fueron desgastando el proceso.

En la experiencia reflexiva surge la necesidad de incorporar elementos pedagógicos para la gestación y construcción de espacios colectivos. Cuando dichos espacios son horizontales, es compleja la definición y explicitación de los propósitos. No hubo objetivos forjados en conjunto explícitamente. Si bien cada colectivo tenía los suyos, no logramos generar las condiciones para dar lugar a este proceso. El trabajo se desarrolló mayormente en base a los adoptados por cada colectivo, surgiendo dificultades en relación con su carácter de tácitos a efecto de los acuerdos grupales y su puesta en práctica. Esto dificulta también la síntesis 
colectiva, partiendo de la base de que dicha síntesis ha de hacerse en función de los objetivos forjados.

Sin embargo, los mundos de vida se entretejieron, revalorizando la riqueza del encuentro y el trabajo. En la olla, se mezclaba la cocción de alimentos orgánicos de nuestras huertas y la huerta de la Unidad 6 del INR, autogestionada en forma cooperativa por los compañeros PLA, nombrada Tupínambá o "Los primeros": nombre de una etnia tribal del Brasil que resiste el exterminio. Los alcances del entramado realizado en conjunto entre todas las personas involucradas en la práctica extensionista, será la cosecha del aprendizaje concreto e intersubjetivo, desde el reconocimiento y la resistencia pedagógico-cultural. En este sentido podemos decir, alejándonos del pensamiento resultadista en términos educativos, que se habilitó una visión desde la ecoepistemología sobre la cual se pudo desarrollar la práctica extensionista que también repercutió en el CeRP y el resto de nuestros espacios.

A la luz de estos aprendizajes se pueden tomar decisiones para el "día después", que es el día en que las personas PLA son devueltas a la calle, a la libertad ambulatoria, para todos quienes participan del encuentro. Podemos posicionarnos desde otros lugares, acompañando, compartiendo, y entablando espacios de diálogo que permiten visibilizar futuros posibles.

Si bien consideramos que la extensión debe institucionalizarse, es importante que al efectivizarse esta no pierda su autenticidad, convirtiéndose en una práctica vacía alejada del compromiso con el medio. Instituir el vínculo recíproco y horizontal de saberes entre el centro educativo de formación del profesorado y el resto de la sociedad.

Entendemos la construcción de la práctica extensionista en formación del profesorado como un espacio educativo no mercantilizable, reivindicando la educación pública, en el camino hacia una universidad de la educación. La extensión y la alteridad en la práctica pedagógica ecocrítica en clave horizontal, superando las dificultades y desencuentros, habilitan la cocreación de los órdenes estudiantil y docente de la currícula de formación profesional desde una destacada autonomía de la participación del estudiantado.

Respecto a las personas PLA, protagonistas del proceso, recuperamos el análisis que hacen sobre el momento al cual acceden a la educación, derecho humano fundamental, la cual sienten como "media libertad". La idea nos interpela sobre el supuesto implícito de la situación de privación de libertad ambulatoria como una supresión de su calidad de sujeto de derechos. Se presenta una demostración moral de lo que significa la pena: castigo. Aparece la dicotomía entre lo escrito en derechos y lo que se vive dentro de determinados extractos sociales, en una sociedad fragmentada que sustenta los privilegios de algunos en el despojo de la mayoría. Cuando los derechos son sólo declarados, la educación se transforma en privilegio.

Los vínculos personales se hacen presente en la calle. Nos conocimos trabajando en la cárcel y luego se hace visible la presencia de un liberado en el mismo entorno. Los actores nos reconocemos, nos alegramos del encuentro, dialogamos y reforzamos la idea de reconocimiento colectivo. En contraposición, la 
problemática inherente a esta sociedad capitalista de producción y consumo en que la mayoría de la población no participa de la apropiación de la riqueza o ni siquiera del proceso productivo, y que por otro lado propone como metas el éxito y el consumo indiscriminado, se presenta como la dificultad para acceder justamente a eso que se propone. La mercantilización de las personas, los conocimientos, los derechos y las cosas es sostenida gracias a la producción social con fuertes limitaciones a su acceso. Este hecho lo vimos reflejado claramente en los contextos de encierro, habitados por personas que nacen y viven en situación de pobreza y que en muchos casos están ahí por haber quedado excluidos del sistema productivo y de consumo. Por otra parte, dentro de dichos contextos lo que se les ofrece sigue las mismas lógicas del sistema capitalista llevadas al extremo por la ausencia de derechos y garantías mínimas para las personas trabajadoras.

Entendemos que la recopilación de la experiencia sirve de guía para muchas personas que trabajan como docentes, que piensan y cada día luchan por convertir en sueño, el anhelo de una educación innovadora, socialmente equitativa, culturalmente poderosa y totalmente libre (Carbonell, 2015). Consideramos de vital importancia para el futuro de la educación apostar por miradas alternativas hacia aquellas cuestiones que nos apelan de manera directa como profesionales de la educación y como humanidad.

\section{Referencias}

Carbonell, J. (2015). Pedagogías del siglo XXI. Barcelona, España: Octaedro.

Castro, J. (2007 [1941]). El banco fijo y la mesa colectiva. Vieja y nueva educación. Montevideo, Uruguay: MEC.

Chevallard, Y. (1998). La transposición didáctica. Del saber sabio al saber enseñado. 3. ed. Buenos Aires, Argentina: Aique.

de Sousa Santos, B. (2010). Descolonizar el saber, reinventar el poder. Montevideo, Uruguay: Trilce.

Fernández, S. y Goicoechea, M. (2011) La educación en contextos de encierro. Instituto de Profesores Artigas, Montevideo. Recuperado en 2020: http://ipa.cfe.edu.uy/images/materiales/inves_educ/laura_abero/educacio n_contexto_encierro.pdf

Foucault, M. (2002[1975]). Vigilar y castigar: nacimiento de la prisión. Buenos Aires, Argentina: Siglo XXI.

Litwin, E. (1997). Las configuraciones didácticas. Una nueva agenda para la enseñanza. Buenos Aires, Argentina: Paidós.

Machado, A. (1912). Campos de Castilla. Proverbios y Cantares. s/d. 
Medina, J.M. y Tommasino, H. (Comps.) (2018). Extensión crítica. Construcción de una Universidad en contexto. Rosario, Argentina: UNR.

Pérez, C. (2016). Contexto de encierro y educación: obstáculos y facilitadores desde una experiencia de asesoría pedagógica. (Tesis de Grado). Facultad de Psicología, UdelaR, Montevideo. Recuperado de: https://sifp.psico.edu.uy/sites/default/files/Trabajos\%20finales/\%20Arch ivos/tfg_camila_perez_version_final.pdf

Ramírez, A. B. (2017). La cartografía pedagógica: un lente para investigar las experiencias educativas. Revista Internacional Magisterio, (82). Recuperado de:

https://www.magisterio.com.co/articulo/la-cartografia-pedagogica-un-lent e-para-investigar-las-experiencias-educativas\#: : :text=y $\% 20$ Amador $\% 2 \mathrm{C} \%$ 20I.C.\%20(2014),127\%2D141.

Salvá, N. (2011). La Educación Superior en el contexto actual. Ausencias, presencias y emergencias. Revista Voces, Montevideo, Año XIV (36), pp.10-22.

Segato, R. (2014). El sexo y la norma: frente estatal, desposesión, patriarcado, colonidad. Revista Estudios feministas, 22 (2), pp.593-616.

Wacqüant, L. (1999). Las cárceles de la miseria. Buenos Aires, Argentina: Manantial. 